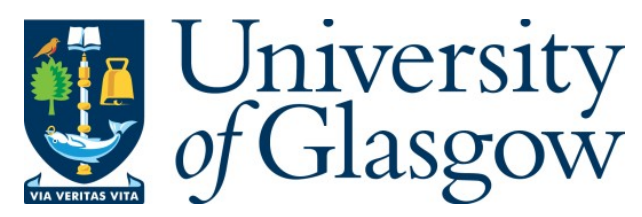

Aguayo, S., Strange, A., Gadegaard, N., Dalby, M.J., and Bozec, L. (2016)

Influence of biomaterial nanotopography on the adhesive and elastic properties of Staphylococcus aureus cells. RSC Advances, 6(92), pp. 89347-89355.

There may be differences between this version and the published version. You are advised to consult the publisher's version if you wish to cite from it.

http://eprints.gla.ac.uk/131308/

Deposited on: 10 January 2017

Enlighten - Research publications by members of the University of Glasgow http://eprints.gla.ac.uk 


\section{Influence of biomaterial nanotopography on the adhesive and elastic properties of Staphylococcus aureus cells}

S Aguayo* $^{*}$, A Strange ${ }^{1}$, N Gadegaard ${ }^{2}$, MJ Dalby ${ }^{3}$, L Bozec $^{1}$

${ }^{1}$ Department of Biomaterials and Tissue Engineering, UCL Eastman Dental Institute, University College London, London, UK

2 Division of Biomedical Engineering, School of Engineering, University of Glasgow, UK ${ }^{3}$ Centre for Cell Engineering; Institute of Molecular, Cell and Systems Biology,

University of Glasgow, UK

\section{Corresponding author:}

* Sebastian Aguayo

Division of Biomaterials and Tissue Engineering

UCL Eastman Dental Institute

University College London

256 Gray's Inn Road, London

WC1X 8LD - UK

E: Sebastian.aguayo.13@ucl.ac.uk 


\begin{abstract}
:
Despite the well-known beneficial effects of biomaterial nanopatterning on host tissue integration, the influence of controlled nanoscale topography on bacterial colonisation and infection remains unknown. Therefore, the aim of the present study was to determine the nanoscale effect of surface nanopatterning on biomaterial colonisation by S. aureus, utilising AFM nanomechanics and single-cell force spectroscopy (SCFS). Nanoindentation of S. aureus bound to planar (PL) and nanopatterned (SQ) polycarbonate (PC) surfaces suggested two distinct areas of mechanical properties, consistent with a central bacterial cell surrounded by a capsullar component. Nevertheless, no differences in elastic moduli were found between bacteria bound to PL and SQ, suggesting a minor role of nanopatterning in bacterial cell elasticity. Furthermore, SCFS demonstrated increased adhesion forces and work between S. aureus and SQ surfaces at 0 s and 1s contact times. Although WLC modelling showed similarities in contour lengths for attachment to both surfaces, Poisson analysis suggests increased short-range forces for the S. aureus-SQ interactions. In the case of $S$. aureus-PL, long-range forces were found to not only be dominant but also repulsive in nature, which may help explain the reduced adhesion forces observed during AFM probing. In conclusion, although surface nanopatterning does not significantly influence the elasticity of attached bacterial cells, it was found to promote the early-adhesion of $S$. aureus cells to the biomaterial surface.
\end{abstract}




\section{Introduction:}

Biomaterials are currently being employed in modern medicine for the augmentation or replacement of missing or diseased tissues ${ }^{1,2}$. More specifically, they are used in a wide range of medical applications such as catheters, artificial heart valve replacements, and orthopaedic and dental implants ${ }^{3}$. In recent years, many surface modifications have been incorporated into the design of biomaterials in the hope of improving their biological activity and host tissue integration ${ }^{3,4}$. Amongst these improvements, controlled nanopatterning of the biomaterial surface has been shown to directly influence human stem cell proliferation and differentiation, giving it an important advantage compared to uncontrolled topographies and planar surfaces ${ }^{5,6}$. Despite these positive effects, the effect of biomaterial nanopatterning on bacterial surface colonisation remains unknown?7.

After implantation, many bacterial strains have demonstrated an increased capacity of adhering to the surface of biomaterials and artificial implants ${ }^{8}$. As implant surface infection has been repeatedly shown to be detrimental for biomaterial-host tissue integration and can lead to complications as severe as replacement surgery, there is current focus on understanding the process of bacterial adhesion to biomaterial surfaces ${ }^{9}$. Amongst others, Staphylococcus aureus has shown an increased likelihood to colonise and infect implants in humans and its presence is related to negative clinical prognosis ${ }^{10-12}$. Furthermore, in recent years S. aureus has demonstrated increased antibiotic resistance and as a result, novel antibacterial and anti-adhesive approaches are currently being explored in hopes of developing new strategies against biomaterial surface infection ${ }^{13-15}$. 
Atomic force microscopy (AFM) has proven to be a valuable tool for the invitro characterisation of cellular and sub-cellular mechanics ${ }^{16}$. Particularly for microbiology, it allows the possibility to study the nanomechanics of living bacterial cells in buffer conditions without the need for prior sample preparation ${ }^{17}$. Using this methodology, it is possible to assess the elastic properties of surface-bound bacteria by indenting the bacterial surface with an AFM cantilever ${ }^{18}$. Furthermore, by employing approaches such as single-cell force spectroscopy (SCFS), bacterial adhesion to biological and non-biological surfaces can be studied in the nano- and pico-meter ranges ${ }^{19-21}$.

Several studies have used AFM to probe the nanomechanics of S. aureus and its adhesion to substrates and other cells ${ }^{22-24}$. However, little is known regarding the influence that nanopatterning exerts on S. aureus adhesion and early-colonisation of the implant surface. As a result, the aim of this study was to determine the nanoscale effect of surface nanopatterning on S. aureus biomaterial colonisation by utilising AFM nanoindentation and single-cell force spectroscopy (SCFS) techniques. 


\section{Experimental:}

\subsection{Polycarbonate surface characterisation}

Two distinct engineered polycarbonate (PC) surfaces were employed throughout the study. Nanopatterned PC surfaces, consisting of $120 \mathrm{~nm}$ pits with $300 \mathrm{~nm}$ centre-centre separation in a square arrangement (SQ), were obtained with a previously reported protocol ${ }^{5}$. A planar PC surface (PL) was employed as a smooth control.

Previous to any measurements, nanopatterned surfaces were prepared and cleaned by sonication in $\mathrm{dH}_{2} \mathrm{O}$ for 5 mins, washed with $70 \%$ ethanol and dried under $\mathrm{N}_{2}$ airflow. Characterisation of surface topography was obtained by AFM imaging (Dimension 3100, Bruker, Santa Barbara, USA) employing intermittent contact mode in air, utilising MSNL-10 (Bruker, USA) cantilevers with a scanning rate of 1.0Hz. Average surface roughness $\left(\mathrm{R}_{\mathrm{a}}\right)$ was determined using height images obtained during AFM scanning $(\mathrm{n}=3)$, and processed using proprietary NanoScope Analysis 1.5 software (Bruker, USA). Surface hydrophilicity was determined by contact angle measurements with deionised water $\left(\mathrm{dH}_{2} \mathrm{O}\right)$ utilising a Cam 200 Optical Contact Angle Meter (Biolin Scientific, Germany). A single $5 \mu l$ droplet was applied to the surfaces, and the average angle of contact over 10 seconds was measured and recorded $(n=3)$. For bacterial experiments, surfaces were placed in a UV-chamber and sterilised with a 20min cycle (BR-506, UVC Light Products, UK).

\subsection{Bacterial cultures}

Stocks of S. aureus (strain 8325-4) were maintained at $-80^{\circ} \mathrm{C}$ in $85 \%$ glycerol/15\% tryto-soy broth (TSB, Oxoid Ltd, UK) medium. For experiments, $S$. 
aureus were grown in TSB for $16 \mathrm{hrs}$ at $37^{\circ} \mathrm{C}$ and aeration until stationary phase. Subsequently, $100 \mu$ of bacterial suspension was diluted 10 -fold in phosphate-buffer saline (PBS 1x, Lonza, Belgium) and harvested at 5000rpm for 1min (Eppendorf 5417R, UK). Resulting pellets were re-suspended in $1 \mathrm{~mL}$ PBS and utilised immediately for experiments.

\subsection{Sample preparation for scanning electron microscopy/focused ion beam (SEM-FIB) milling and imaging.}

To obtain images of S. aureus colonisation of PC surfaces, a $500 \mu$ d droplet of bacterial suspension was incubated on each surface for $10 \mathrm{~min}$, rinsed with PBS to remove unattached cells, and fixed immediately with $4 \%$ glutaraldehyde. Samples were then dehydrated with 10 min serial washes in 50, 70, 90 and $100 \%$ ethanol, and sputter coated with gold. Imaging was carried out with an XB1540 (Carl Zeiss, Germany) SEM-FIB system with an acceleration voltage of $10 \mathrm{kV}$ at magnifications of 50,000x and 100,000x. FIB milling was carried out with a 30kV:20mA gallium beam probe, by tilting the sample $54^{\circ}$ and performing serial linear millings on $S$. aureus cells until exposing the bacterial-surface interface.

\subsection{Sample preparation for AFM}

\subsubsection{AFM imaging and bacterial nanomechanics}

To attach single $S$. aureus cells onto substrates for imaging and nanomechanics, a $20 \mu \mathrm{l}$ droplet of bacterial suspension was deposited onto each PC surface and incubated for $10 \mathrm{~min}$. For imaging, samples were washed after 
incubation with $\mathrm{dH}_{2} \mathrm{O}$ and softly dried under $\mathrm{N}_{2}$ airflow. For nanoindentation, samples were washed with PBS to remove unattached cells, and re-suspended with $100 \mu$ l of TRIS buffer (Sigma-Aldrich, UK). For all nanomechanic experiments, bacterial cells were maintained submerged in TRIS buffer throughout experimentation.

Both imaging and force-volume mapping of the bacterial surface of S. aureus were obtained by employing a JPK Nanowizard system (JPK Instruments, Germany) mounted on an inverted optical microscope (Olympus IX71, Olympus, Japan). Imaging was carried out with a NCS35 cantilever (MikroMasch, USA) in intermittent contact mode in air, tuned at $\sim 110 \mathrm{KHz}$. Set point and gain values were adjusted insitu during scanning for image optimisation. Images were obtained at $512 \times 512$ pixels with an average scanning rate of $0.5 \mathrm{~Hz}$.

For force-volume mapping, MSNL-10 cantilevers with a spring constant of $\sim 0.1 \mathrm{~N} / \mathrm{m}$ were used. After locating an isolated attached bacterium, force-curves were obtained (constant speed of $2 \mu \mathrm{m} / \mathrm{s}$ and a loading force of $3 \mathrm{nN}$ ) at random points of the cell centre and perimeter of each bacterium. Six independent S. aureus cells were indented and analysed per surface. As minimal adhesion between the cantilever and bacterial cell was recorded and an indentation depth of $\sim 50 \mathrm{~nm}$ was obtained, Young's modulus (YM) was determined from the extension curve by applying the Hertzian model as previously described in the literature 25 :

$$
F=\frac{4 E R^{1 / 2} \delta^{3 / 2}}{3\left(1-v^{2}\right)}
$$


where $\mathrm{F}$ is force, $\mathrm{E}$ is the Young's modulus, $\mathrm{R}$ is the radius of the indenter, $\delta$ is indentation distance and $\mathrm{v}$ is the Poisson's ratio of the indented sample (considered as 0.5$)$.

\subsubsection{Single-cell force spectroscopy (SCFS)}

For SCFS experiments, customised colloidal probes were fabricated in order to immobilise S. aureus cells by utilising a protocol previously published ${ }^{23}$. Briefly, the end of a tipless cantilever (NP-010, Bruker, Santa Barbara, USA) was brought into contact with a thin layer of UV-curable glue (Loctite, UK) for 10s. Subsequently, the glue-coated tip was approached to a $\sim 10 \mu \mathrm{m}$ silica microsphere (Whitehouse Scientific, UK) with a maximum loading force of $0.5 \mathrm{nN}$ for $3 \mathrm{~min}$. Upon retraction, effective attachment of the silica bead was observed by optical microscopy. Functionalised cantilevers were then UV-cured for $10 \mathrm{mins}$, and correct placing of the microsphere was assessed by SEM imaging (Philips XL30 FEG SEM, FEI, Eindhoven, Netherlands). As a next step, functionalised cantilevers were coated for $1 \mathrm{hr}$ with a solution of $4 \mathrm{mg} / \mathrm{ml}$ dopamine hydrochloride (poly-DOPA) in 10mM TRIS buffer (pH8.0), washed with $\mathrm{dH}_{2} \mathrm{O}$ and dried with $\mathrm{N}_{2}$. All cantilevers were calibrated using thermal tuning $\left(\sim 0.3 \mathrm{~N} / \mathrm{m}\right.$ spring constants), and stored at $4^{\circ} \mathrm{C}$ until $\mathrm{AFM}$ experiments.

To functionalise colloidal probes with living S. aureus cells, cantilevers were mounted onto the AFM and submerged into a $20 \mu$ d droplet of bacterial suspension. The probe was then brought into contact with an isolated cell, with a loading force of $0.5 \mathrm{nN}$ for $\sim 3 \mathrm{mins}$ until attachment was observed. Cantilevers were then retracted, transferred above the PC surface and submerged in TRIS buffer being 
careful not to dehydrate the S. aureus probe. Experiments were carried out with a loading force of $0.5 \mathrm{nN}$, a constant speed of $2 \mu \mathrm{m} / \mathrm{s}$, and surface delay times of $0 \mathrm{~s}$ and 1s. Each of the $S$. aureus functionalised probes were utilised only for a single experiment and discarded thereafter. Four independent S. aureus probes were utilised for each surface (totalling 8 probes).

\subsection{Data analysis:}

All obtained images and force curves were analysed using the JPK Data Processing Software v.5.1.8 (JPK Instruments, Germany). For nanomechanics, histograms and median (Mdn) values were obtained for each surface and contact time, and significance was determined by applying the non-parametric MannWhitney test $(\mathrm{p}<0.05)$. From SCFS force curves, maximum adhesion force $(\mathrm{nN})$ between the bacterial cell and cantilever was obtained as the lowest negative value for force during the retraction phase, and overall adhesion work (aJ) was obtained by integrating the area under the retraction curve. Unbinding peaks observed during retraction were fitted with the worm-like chain (WLC) model as previously described assuming a persistent length of $0.36 \mathrm{~nm}^{26}$. Finally, a Poisson analysis of $S$. aureus-PC unbinding was performed employing a previously published approach, to decouple adhesion into short-range $\left(\mathrm{F}_{\mathrm{SR}}\right)$ and long-range $\left(\mathrm{F}_{\mathrm{LR}}\right)$ forces ${ }^{27}$. 


\section{Results and discussion:}

\subsection{Characterisation of bacterial adhesion onto PC surfaces}

AFM imaging of PC substrates demonstrated different topographies for the PL and SQ surfaces (Figure 1). SQ exhibited a very distinct patterning, with clearly defined nanopits at regular intervals consistently throughout the surface, which contrasted strongly with the smooth topography observed for PL. AFM surface cross sections of SQ further showed rounded nanopits with an average diameter of $99 \pm$ $6 \mathrm{~nm}(\mathrm{n}=20)$ and a depth of $\sim 70 \mathrm{~nm}$. Also, $\mathrm{R}_{\mathrm{a}}$ measurements showed an increased surface roughness of $13.7 \pm 0.8 \mathrm{~nm}$ for $\mathrm{SQ}$ surfaces compared to $0.4 \pm 0.0 \mathrm{~nm}$ for PL ones. No difference was found in surface wettability, with values for PL and SQ at $80.6 \pm 2.7^{\circ}$ and $80.3 \pm 1.5^{\circ}$ respectively. However these values do indicate that PC surfaces, irrespective of patterning, are slightly hydrophilic which has been previously shown to favour staphylococcal attachment ${ }^{28}$.

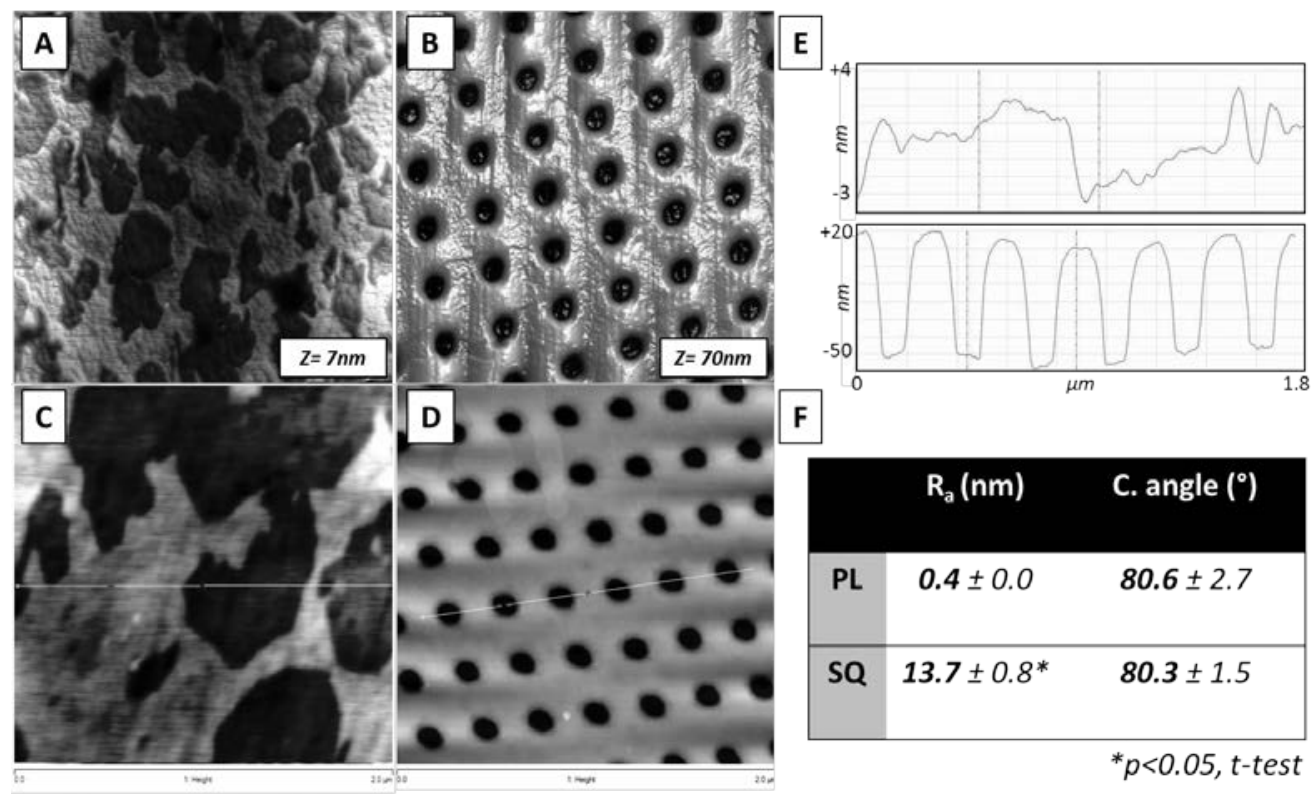


Figure 1: Characterisation of planar (PL) and nanopatterned (SQ) polycarbonate (PC) surfaces. $2 \times 2 \mu \mathrm{m}$ AFM 3D reconstruction images of (A) PL and (B) SQ demonstrate marked topographical differences between both surfaces. (C) and (D) correspond to AFM height scans for PL and SQ respectively, from which surface cross sections were obtained (E) ( $2 \times 2 \mu \mathrm{m}$ scans). From these cross sections, it is possible to observe nanopit depth and patterning on the SQ surface. (F) Although surface roughness was increased in SQ compared to PL surfaces, no differences were found in regards to surface wettability as both substrates were found to display a slightly hydrophilic behaviour.

Images of surface-bound $S$. aureus cells were successfully obtained with intermittent contact mode AFM imaging. More specifically, it was possible to observe the $S$. aureus cell surrounded by a microcapsule (Figure 2.a and b). This area appears to not only surround the bacterial cell but to also cover its surface partially, consistent with previous AFM observations which employed a similar $S$. aureus strain ${ }^{29}$. Further phase contrast imaging, which has been previously used to differentiate cell from capsule in streptococcal species ${ }^{30}$, was used to demonstrate differences in physico-mechanical properties between the bacterial cell and adjacent area (Figure 2.c). Height images obtained with force-volume mapping in buffer are also consistent with this bacterial morphology, and although resolution is not as high as with intermittent contact imaging, the bacteria-capsule structure can still be clearly observed in the corresponding pixel map (Figure 4.a). Interestingly, previous research suggests that although S. aureus strain 8325-4 carries a serotype5 capsule gene, it is defective in capsule expression ${ }^{31,32}$. However, Coldren and colleagues found very similar morphological characteristics in a serotype-8 capsulepositive $S$, aureus strain when imaged with AFM under comparable conditions ${ }^{33}$. 
Serotype- 5 and serotype- 8 bacterial capsules are considered to have similar characteristics ${ }^{31}$, and therefore it is possible that the strain is effectively expressing a capsule-like structure.

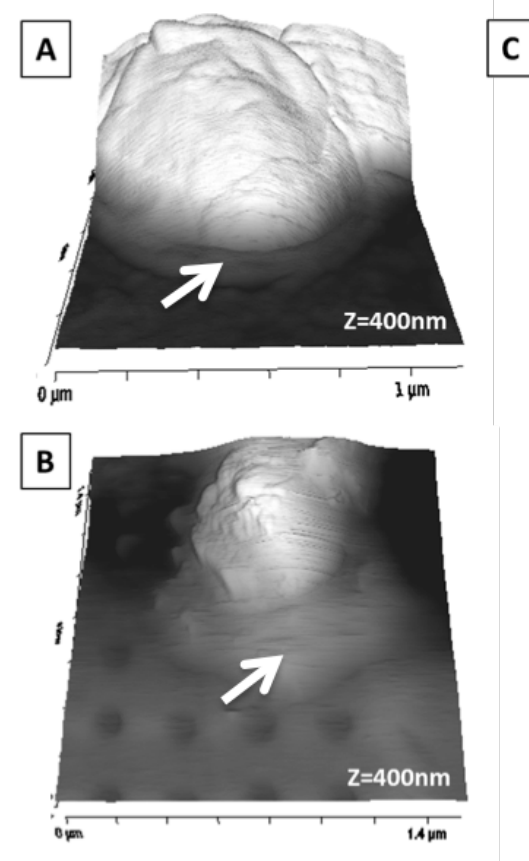

C

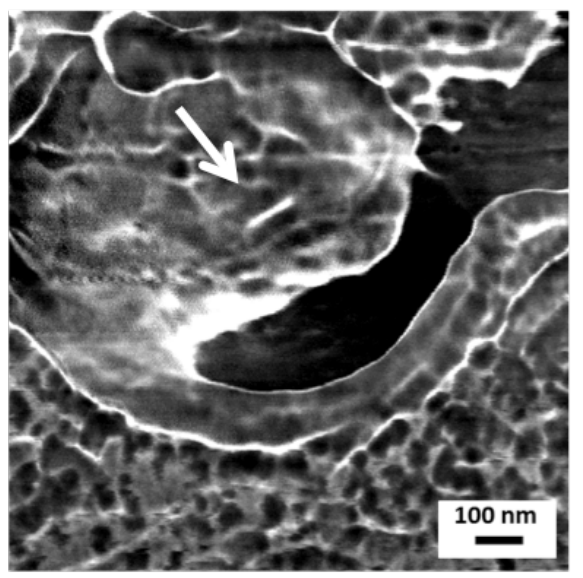

Figure 2: AFM intermittent contact imaging of $S$. aureus 8325-4 adhered to PL and SQ surfaces. 3D reconstruction images of S. aureus attached to (A) PL and (B) SQ surfaces. It is possible to observe the S. aureus cell surrounded and partially covered by capsule (inset arrows) (C) Phase contrast image obtained for the bacterium imaged in (A), which evidences distinct structural composition for both the S. aureus cell and capsule (arrow). 


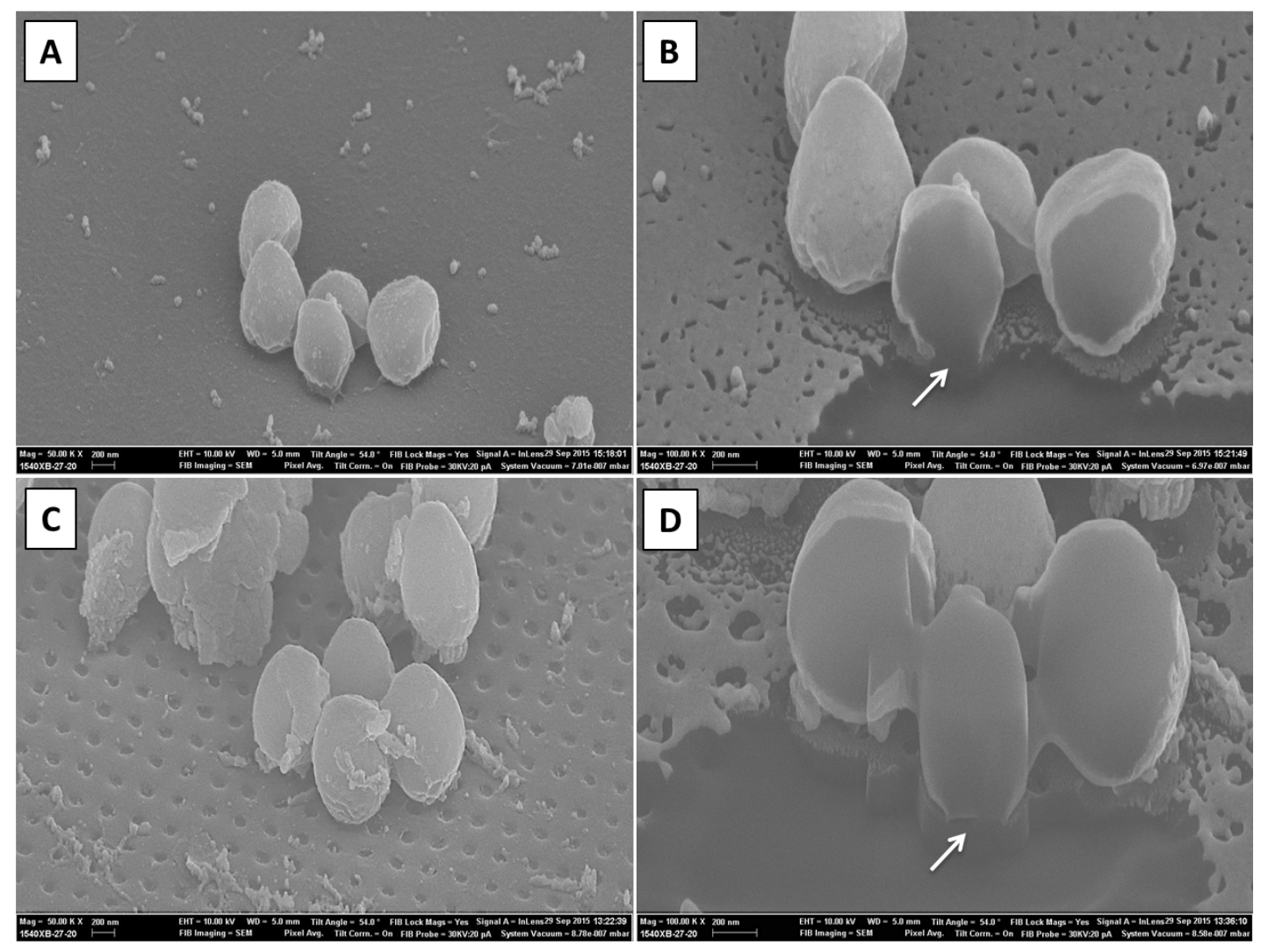

Figure 3: SEM-FIB imaging and milling of the S. aureus-PC interface. Imaging of S. aureus cells attached to PL (A, B) and SQ (C, D) surfaces before and after FIB milling, respectively (A and C 50,000x; B and D 100,000x). S. aureus capsule is absent due to it being destroyed during sample preparation, nevertheless, a minor degree of interaction can still be observed between bacterial cells and PC surfaces after FIB milling. 
A

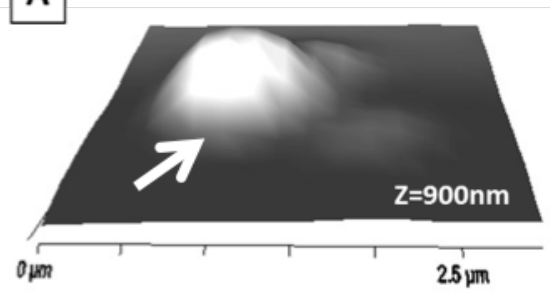

c

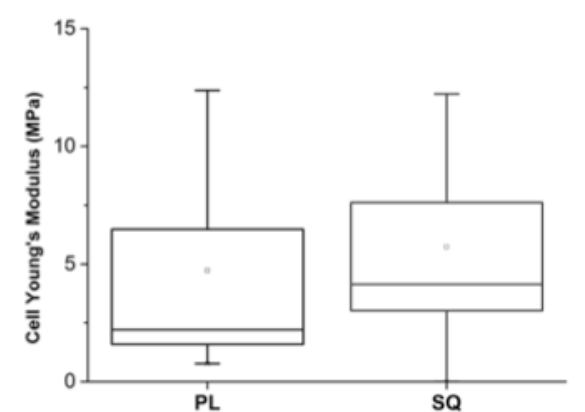

B
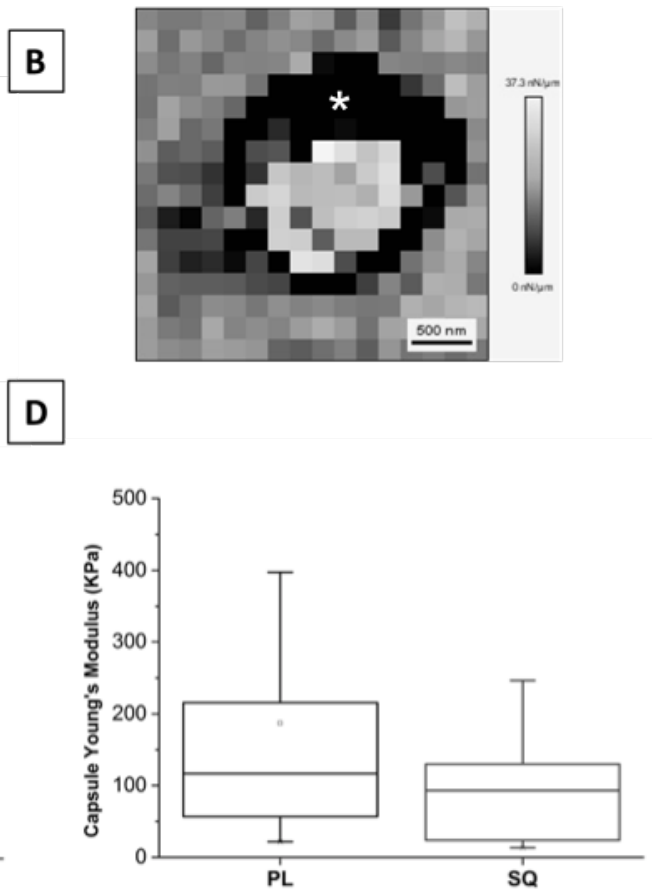

Figure 4: Nanomechanics of surface-bound $S$. aureus cells attached to PL and SQ in buffer. (A)

3D reconstruction image obtained during a $S$. aureus force-volume map, in which both the bacterial cell and capsule (arrow) can be observed. (B) 16x16pixel stiffness map on the surface of a S. aureus cell attached to a PL surface. An area of decreased stiffness can be observed surrounding the bacterial cell (asterisk). Values for Young's modulus (YM) obtained with the Hertzian model for (C) the bacterial cell and (D) capsule are shown ( $\mathrm{n}=6$ independent cells). A marked difference is observed between the two regions, with the bacterial cell showing values in the MPa range and capsule being in the $\mathrm{kPa}$ range. No significant differences in YM were found between PL and SQ suggesting that surface nanotopography does not influence the mechanics of $S$. aureus cells $(\mathrm{p}>0.05$, Mann-Whitney).

Although careful preparation was used, it was not possible to image the $S$. aureus capsule under the SEM-FIB, suggesting that it is destroyed or lost during sample dehydration and preparation (Figure 3). Subsequently, upon exposing the S. aureusPC interface with FIB milling, only minor contact between bacteria and surface 
could be observed and therefore it is believed that the bacteria-substrate interaction is mostly mediated by capsule rather than by the bacterial cell itself. Overall, imaging suggests that $S$. aureus capsule does not only account for a significant part of bacterial size observed in AFM imaging, but most importantly, it also increases the effective contact area between the bacterium and PC surface during surface attachment.

\subsection{Underlying substrate topography does not influence the nanomechanical properties of surface-bound $S$. aureus}

The elastic properties of surface-bound S. aureus were obtained in force-volume mode by performing a number of force curves on the bacterial surface. Two distinct mechanical behaviours were observed for S. aureus, consistent with the presence of a stiffer cell body surrounded by capsule of decreased stiffness (Figure 4.b). This observation was consistent throughout measurements for $S$. aureus cells attached to both studied PC surfaces. YM for the bacterial cell was found to be 2.20MPa for PL and 4.14MPa for $\mathrm{SQ}$, in the range of previously reported values for S. aureus 8325-4 elasticity ${ }^{34}$. However, surrounding the central bacterium, an area with significantly reduced $\mathrm{YM}$ of $116.58 \mathrm{kPa}$ for $\mathrm{PL}$ and $92.89 \mathrm{kPa}$ for $\mathrm{SQ}$ was observed $(\mathrm{p}<0.05)$ (Figure 4.c and d).

Regarding the influence of the underlying surface on of S. aureus nanomechanics, no significant differences were found in YM between bacteria bound to PL and SQ substrates ( $p>0.05)$ (Figure 4.c and d). As AFM imaging demonstrated that a typical S. aureus cell directly interacts with a number of nanopits on the SQ surface, any 
effect that nanotopography may have on bacterial cell elasticity should be clearly noticeable at the single-cell level. However, both $S$. aureus cell and capsule nanomechanics were not affected by the presence or absence of surface nanopatterning. It is also possible that nanopatterning may only exert a localised effect on elasticity in the vicinity of the bacteria-nanopattern interface, and therefore it cannot be explored by solely indenting the top region of attached $S$. aureus cells.

\subsection{Adhesion forces between $S$, aureus-PC surfaces are increased by the presence of surface nanopatterning}

To analyse the effect of surface nanopatterning on the early colonisation of $S$. aureus, functionalised AFM bacterial probes were constructed and probed against PC surfaces at 0 s and 1 s contact times (Figures 5 and 6). Adhesion force and work between S. aureus and PL surfaces were found to be $<0.05 \mathrm{nN}$ and $<0.05 \mathrm{aJ}$ at $0 \mathrm{~s}$ surface contact times delays. However, increasing the contact time to $1 \mathrm{~s}$ raised these values to $0.11 \mathrm{nN}$ and $5.01 \mathrm{a}$ ) respectively $(\mathrm{p}<0.0001)$. Adhesion forces between $S$. aureus and SQ surfaces was increased at both time points compared to PL, with values of $0.10 \mathrm{nN}$ at $0 \mathrm{~s}$ and $0.23 \mathrm{nN}$ at $1 \mathrm{~s}$ surface delay times $(\mathrm{p}<0.0001)$. A similar increase was observed for the work of adhesion, with values of 4.28aJ and 18.75aJ for 0 s and 1 s respectively $(\mathrm{p}<0.0001)$. Altogether, these results suggest that both contact time and surface nanopatterning directly influence the early-adhesion of $S$. aureus to PC surfaces. In the literature, SCFS of S. aureus strain 8325-4 has been previously employed to measure its adhesion with Candida albicans hyphae and yeast cells and fibronectin-functionalised AFM cantilevers ${ }^{35,36}$. In both cases, 
reported adhesion forces were of higher magnitudes than the ones observed in the present study between S. aureus and PC. As these studies examined specific receptor-ligand interactions between $S$. aureus and biological substrates, it is possible to hypothesise that the reduced adhesion forces observed for the unbinding of $S$. aureus-PC, irrespective of patterning, is a reflection of a lack of specificity between the bacterial cell and surface. It also remains possible that increased loading forces were employed in these studies, which has been shown to promote bacterial adhesion to substrates ${ }^{37}$.
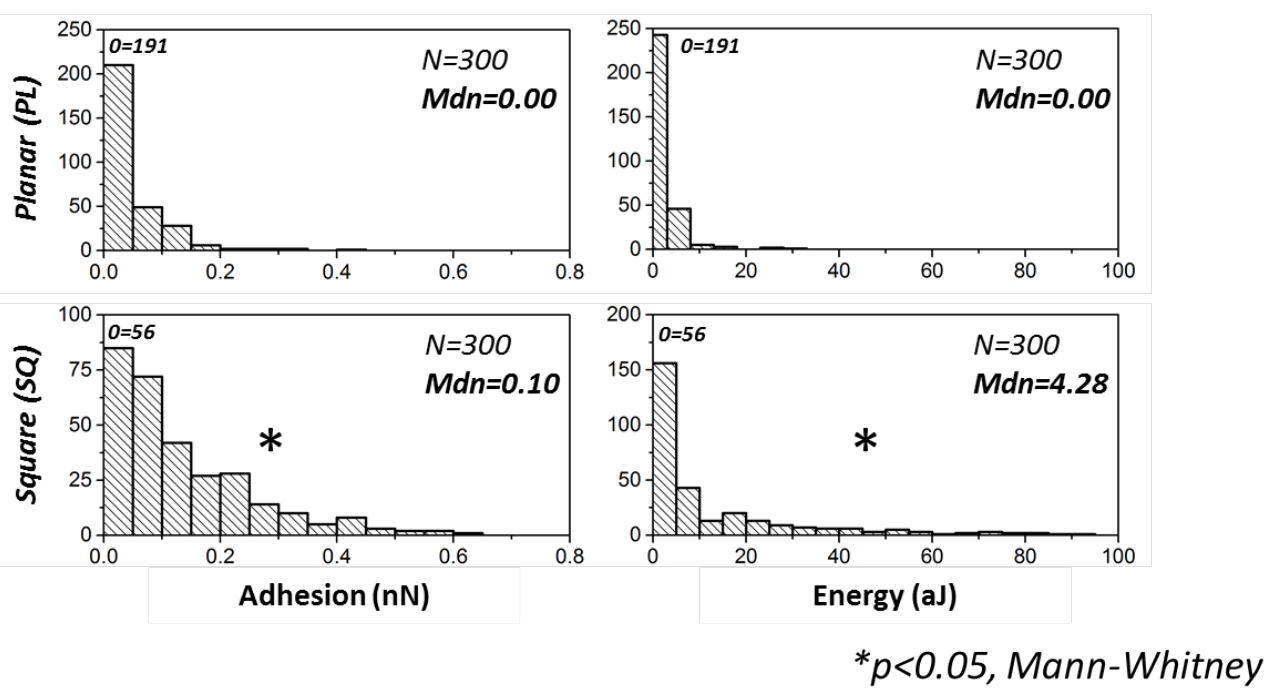

Figure 5: Adhesion forces and work between living $S$. aureus and PC surfaces at short contact times. Histogram of adhesion forces and work recorded between S. aureus-functionalised AFM probes and PL and SQ surfaces, with a surface delay of 0s. For SQ, both parameters were significantly increased compared to PL surfaces $(\mathrm{p}<0.05)$. The number of non-adhesive events per group is indicated in the upper left corner of each histogram. 

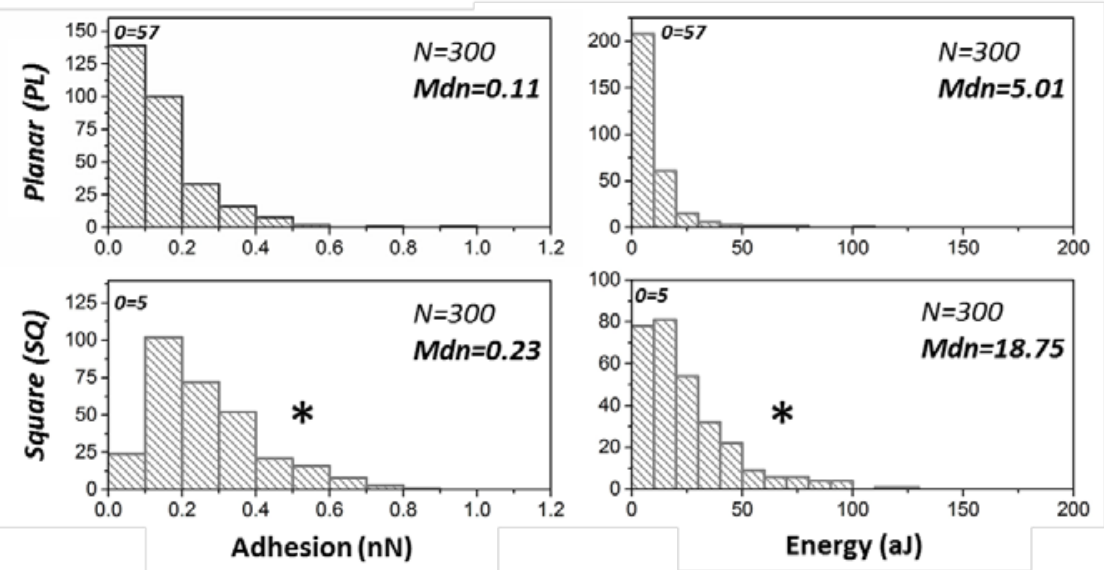

${ }^{*} p<0.05$, Mann-Whitney

Figure 6: Adhesion forces and work between living S. aureus and PC surfaces at increased contact times. Histogram of adhesion forces and work recorded between S. aureus-functionalised AFM probes and PL and SQ surfaces, with a surface delay of 1s. Increasing the contact time to 1s increased adhesion forces and work in both studied surfaces $(\mathrm{p}<0.05)$. Similar to 0 s contact times, both parameters were also found to be increased in SQ compared to PL surfaces $(p<0.05)$.

Representative force curves obtained for both PL and SQ surfaces can be observed in Figure 7.a. In both cases, 'sawtooth-like' unbinding peaks indicative of specific adhesion between the probe and surface were clearly observed throughout measurements. These unbinding events were fitted with the WLC model, yielding contour length values predominantly situated in the 50-400nm range (Figure 7.b). The number of unbinding peaks found for $S Q(n=942)$ was slightly increased compared to PL ( $\mathrm{n}=889)$. Interestingly, it was possible to fit multiple curves to the contour length distributions of both PL and SQ. PL surfaces displayed peaks at $140 \mathrm{~nm}, 270 \mathrm{~nm}$ and $358 \mathrm{~nm}$; while SQ surfaces were also found to have similar peaks 
at lengths of $147 \mathrm{~nm}, 253 \mathrm{~nm}$, and $380 \mathrm{~nm}$ respectively (Figure 7.b). As contact angle measurements demonstrated comparable surface chemistry for both PC surfaces, it is believed that similar bacterial surface receptors are being recruited in S. aureus attachment to PL and SQ. In a recent study, average contour lengths between $S$. aureus and titanium (Ti) implant surfaces were reported at $314.1 \pm 9.3 \mathrm{~nm}^{23}$. Ti is more hydrophilic than PC and biomaterial surface hydrophilicity has been previously shown to favour bacterial adhesion ${ }^{28}$. However, it has yet to be determined if contour lengths observed for S. aureus-PC unbinding correspond to the same Ti-binding receptors stretched to different lengths, or if they reflect another surface adhesion mechanism.
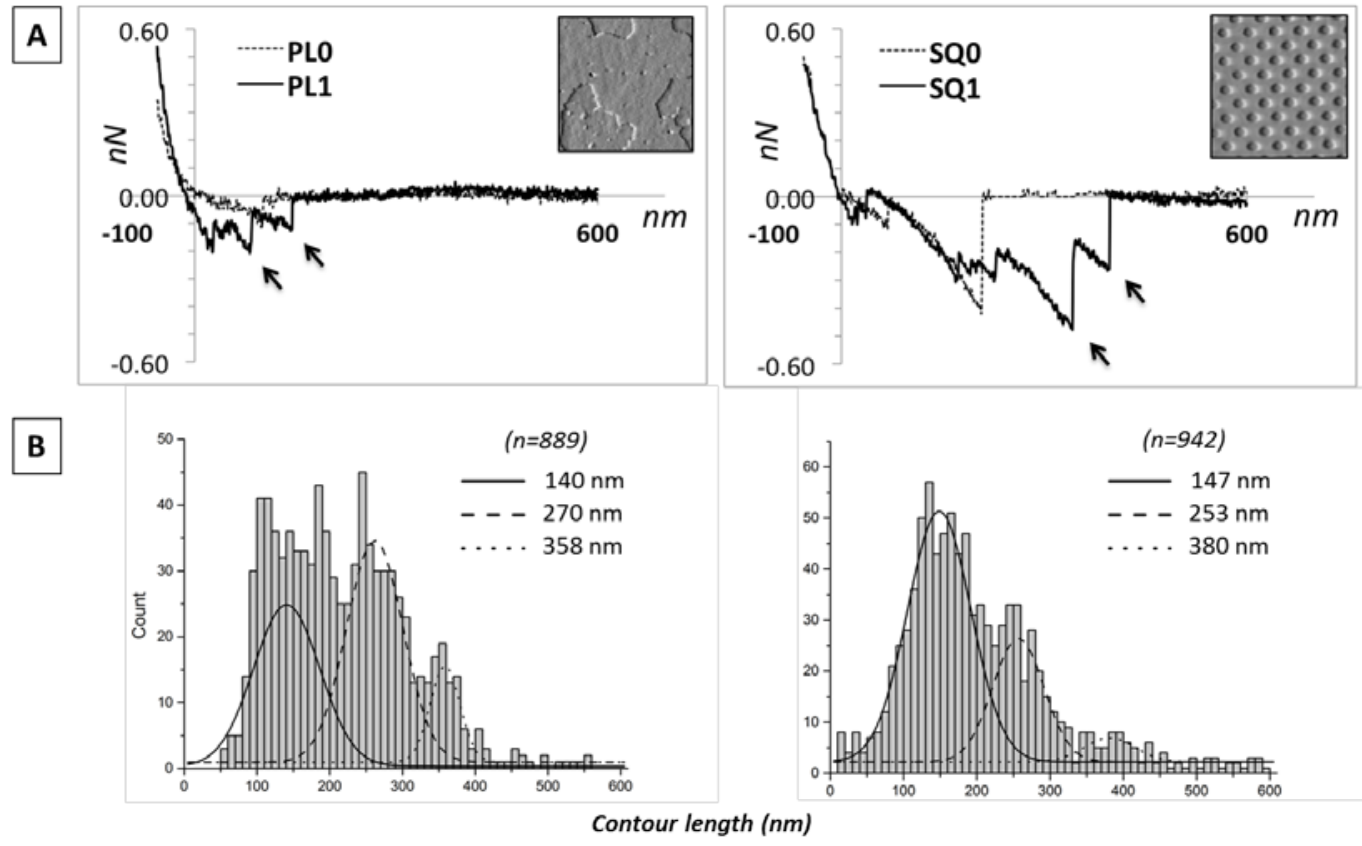

Figure 7: Worm-like chain (WLC) modelling of force-extension peaks observed during $S$. aureus-PC unbinding. (A) Representative retraction curves observed during between S. aureus probes and PL and SQ surfaces. Single unbinding events can be observed in both cases, as indicated by the arrows. Insets represent AFM deflection images of each surface ( $2 x 2 \mu \mathrm{m}$ scans). (B) WLC 
modelling yielded multiple peaks for contour lengths in both PL and SQ, as observed in the corresponding histograms.

Furthermore, Poisson analysis of S. aureus-PC unbinding was carried out to decouple overall adhesion forces into $F_{S R}$ and $F_{L R}$. PL surfaces were found to have a $F_{S R}$ of $-0.08 \pm 0.02 n N$, while $S Q$ surfaces showed an increased value of $-1.42 \pm 0.02 n N$ (Table 1). Interestingly, $F_{L R}$ for PL surfaces was found to be a positive value of $0.38 \pm 0.25 \mathrm{nN}$, and as such it indicates that the overall long-range forces acting between $S$. aureus and PL are repulsive in nature. Increased $\mathrm{F}_{\mathrm{SR}}$ between $S$. aureusSQ surfaces paired with a repulsive $\mathrm{F}_{\mathrm{LR}}$ in $S$. aureus-PL may help explain the reduced adhesion force and work observed in the latter case.

TABLE 1: Poisson analysis of $S$. aureus unbinding from PL and SQ surfaces in buffer

\begin{tabular}{|ccc|cc|}
\hline Surface & \multicolumn{2}{c}{ Specific $\left(\mathbf{F}_{\mathrm{SR}}\right)$} & \multicolumn{2}{c|}{ Non-specific $\left(\mathbf{F}_{\mathbf{L R}}\right)$} \\
\hline & Mean $(n N)$ & SE & Mean $(n N)$ & SE \\
\hline PL & $-\mathbf{0 . 0 8}$ & 0.02 & $\mathbf{0 . 3 8}$ & 0.25 \\
\hline SQ & $-\mathbf{1 . 4 2}$ & 0.02 & $-\mathbf{0 . 2 3}$ & 0.01 \\
\hline
\end{tabular}

Overall, the use of SCFS was an effective approach to study the early-colonisation of S. aureus onto PC surfaces at the nanoscale. In biomaterial infection, early bacterial colonisers are believed to come into contact with the surface with minimal to no external loading forces being applied. Therefore, in this PC-based model of biomaterial nanopatterning, loading forces for $S$. aureus probes were reduced $(0.5 \mathrm{nN})$ to avoid deformation of the bacterial cell during probing. By utilising this 'zero-force contact' approach, it is believed that the influence of AFM probing on 
adhesion values can be kept to a minimum. High loading forces would therefore not effectively recreate the physiological process of bacterial adhesion, as they would be promoting the interaction between bacteria and surface. This effect was recently demonstrated by Chen et al, where the adhesion between S. aureus strain 8325-4 and a glass surface was found to be proportional to the loading force applied ${ }^{37}$.

In the literature, the capsule have been considered to play an active role in bacterial attachment to biomaterial surfaces ${ }^{38,39}$. Considering the early contact times utilised in this study ( 0 and 1 s), it is believed that adhesion between S. aureus 83254 and PL and SQ surfaces at these time points is mostly mediated by the interaction between the capsule and substrate. In the past, surfaces with nanoscale topographies have been found to possess improved antibacterial properties against S. aureus, when bacteria are cultured for $<1 \mathrm{hr}$ and macro-scale bacterial attachment assays such as fluorescence microscopy and spread plate methods were employed ${ }^{7,40-42}$. However, results from the present study suggest that nanopatterning increases the colonisation of biomaterial surfaces at early contact times ( 0 and 1s). Although bacterial adhesion to surfaces is a crucial initial phase ${ }^{43}$, it does not account on its own for the entire process of biomaterial colonisation ${ }^{42}$. Therefore, it remains possible that although attachment of $S$. aureus to nanopatterned surfaces is initially increased at very short time points, bacteria may not be able to effectively colonise the surface due to reduced proliferation capabilities or decreased capsule secretion at increased contact times. Although contact times of $\leq 1 \mathrm{~s}$ may be short in relation to the lifetime of a biomaterial infection process, early-colonising bacteria could potentially become a 'base-layer' 
for the attachment of secondary bacteria at increased time points ${ }^{44}$. Future efforts should focus on further understanding the in-vivo relevance of early biomaterial colonisation by $S$. aureus, and if promoting or inhibiting this initial bacteriumsurface interaction can aid in the search for novel ways to control biofilm formation without compromising the increased biological properties of nanopatterned surfaces. 


\section{Conclusion:}

Both nanoindentation and AFM SCFS were found to be powerful tools to study the nanomechanics of living $S$. aureus cells in buffer conditions. Imaging of surface bound $S$. aureus showed the presence of adjacent capsule regardless of surface patterning, which was found to have reduced elasticity compared to the central bacterial cell. No surface-induced changes in bacterial nanomechanics were found in S. aureus attached to PL and SQ. However, SCFS with S. aureus functionalised probes demonstrated increased adhesion forces and work between bacteria and SQ surfaces. Poisson analysis suggests that this is due to higher short-

range forces between $S$. aureus-SQ and repulsive long-range forces between $S$. aureus-PL. Overall, surface nanotopography was found to influence $S$ aureus attachment to PC surfaces at early time points, and further research should evaluate is this effect is observable at increased contact times and in other biomaterial surfaces of clinical relevance.

\section{Acknowledgements:}

This work has been funded by the Becas-Chile PhD Scholarship Programme. 


\section{References:}

1 Huebsch, N. \& Mooney, D. J. Inspiration and application in the evolution of biomaterials. Nature 462, 426-432, doi:10.1038/nature08601 (2009).

2 Ratner, B. D. \& Bryant, S. J. Biomaterials: where we have been and where we are going. Annu. Rev. Biomed. Eng. 6, 41-75, doi:10.1146/annurev.bioeng.6.040803.140027 (2004).

3 Logan, N. \& Brett, P. The Control of Mesenchymal Stromal Cell Osteogenic Differentiation through Modified Surfaces. Stem cells international 2013, 361637, doi:10.1155/2013/361637 (2013).

4 O'Brien, F. J. Biomaterials \&amp; scaffolds for tissue engineering. Materials Today 14, 88-95, doi:http://dx.doi.org/10.1016/S1369-7021(11)70058-X (2011).

5 Dalby, M. J. et al. The control of human mesenchymal cell differentiation using nanoscale symmetry and disorder. Nature materials 6, 997-1003, doi:10.1038/nmat2013 (2007).

6 Estevez, M., Martinez, E., Yarwood, S. J., Dalby, M. J. \& Samitier, J. Adhesion and migration of cells responding to microtopography. J. Biomed. Mater. Res. A 103, 1659-1668, doi:10.1002/jbm.a.35293 (2015).

7 Jahed, Z. et al. Responses of Staphylococcus aureus bacterial cells to nanocrystalline nickel nanostructures. Biomaterials 35, 4249-4254, doi:10.1016/j.biomaterials.2014.01.080 (2014).

8 Subramani, K., Jung, R. E., Molenberg, A. \& Hammerle, C. H. Biofilm on dental implants: a review of the literature. Int. J. Oral Maxillofac. Implants 24, 616626 (2009).

9 Svensson, S. et al. Role of nanostructured gold surfaces on monocyte activation and Staphylococcus epidermidis biofilm formation. International journal of nanomedicine 9, 775-794, doi:10.2147/ijn.s51465 (2014).

10 Arciola, C. R., Campoccia, D., Speziale, P., Montanaro, L. \& Costerton, J. W. Biofilm formation in Staphylococcus implant infections. A review of molecular mechanisms and implications for biofilm-resistant materials. Biomaterials 33, 5967-5982, doi:10.1016/j.biomaterials.2012.05.031 (2012).

11 Lee, A. \& Wang, H. L. Biofilm related to dental implants. Implant Dent. 19, 387-393, doi:10.1097/ID.0b013e3181effa53 (2010).

12 Harris, L. G., Foster, S. J. \& Richards, R. G. An introduction to Staphylococcus aureus, and techniques for identifying and quantifying $S$. aureus adhesins in relation to adhesion to biomaterials: review. European cells \& materials 4, 3960 (2002).

13 Loskill, P. et al. Reduction of the Peptidoglycan Crosslinking Causes a Decrease in Stiffness of the Staphylococcus aureus Cell Envelope. Biophys. J. 107, 1082-1089, doi:10.1016/j.bpj.2014.07.029 (2014).

14 McKendry, R. A. Nanomechanics of superbugs and superdrugs: new frontiers in nanomedicine. Biochem. Soc. Trans. 40, 603-608, doi:10.1042/BST20120082 (2012). 
15 Francius, G., Domenech, O., Mingeot-Leclercq, M. P. \& Dufrene, Y. F. Direct observation of Staphylococcus aureus cell wall digestion by lysostaphin. J. Bacteriol. 190, 7904-7909, doi:10.1128/JB.01116-08 (2008).

16 Dufrene, Y. F. Sticky microbes: forces in microbial cell adhesion. Trends Microbiol. 23, 376-382, doi:10.1016/j.tim.2015.01.011 (2015).

17 Aguayo, S., Donos, N., Spratt, D. \& Bozec, L. Single-bacterium nanomechanics in biomedicine: unravelling the dynamics of bacterial cells. Nanotechnology 26, 062001, doi:10.1088/0957-4484/26/6/062001 (2015).

18 Webb, H. K., Truong, V. K., Hasan, J., Crawford, R. J. \& Ivanova, E. P. Physicomechanical characterisation of cells using atomic force microscopy - Current research and methodologies. J. Microbiol. Methods 86, 131-139, doi:10.1016/j.mimet.2011.05.021 (2011).

19 Taubenberger, A. V., Hutmacher, D. W. \& Muller, D. J. Single-Cell Force Spectroscopy, an Emerging Tool to Quantify Cell Adhesion to Biomaterials. Tissue Eng Part B Rev, doi:10.1089/ten.TEB.2013.0125 (2013).

20 Beaussart, A. et al. Single-cell force spectroscopy of probiotic bacteria. Biophys. J. 104, 1886-1892, doi:10.1016/j.bpj.2013.03.046 S00063495(13)00385-8 [pii] (2013).

21 Sullan, R. M., Li, J. K., Crowley, P. J., Brady, L. J. \& Dufrene, Y. F. Binding forces of Streptococcus mutans P1 adhesin. ACS Nano 9, 1448-1460, doi:10.1021/nn5058886 (2015).

22 Ovchinnikova, E. S., Krom, B. P., Busscher, H. J. \& van der Mei, H. C. Evaluation of adhesion forces of Staphylococcus aureus along the length of Candida albicans hyphae. BMC Microbiol. 12, 281 (2012).

23 Aguayo, S., Donos, N., Spratt, D. \& Bozec, L. Nanoadhesion of Staphylococcus aureus onto Titanium Implant Surfaces. J. Dent. Res., doi:10.1177/0022034515591485 (2015).

24 Peters, B. M. et al. Staphylococcus aureus adherence to Candida albicans hyphae is mediated by the hyphal adhesin Als3p. Microbiology 158, 29752986 (2012).

25 Formosa, C. et al. Nanoscale analysis of the effects of antibiotics and CX1 on a Pseudomonas aeruginosa multidrug-resistant strain. Sci. Rep. 2, 575, doi:10.1038/srep00575 (2012).

26 Herman, P. et al. The binding force of the staphylococcal adhesin SdrG is remarkably strong. Mol. Microbiol. 93, 356-368, doi:10.1111/mmi.12663 (2014).

27 Chen, Y., Busscher, H. J., van der Mei, H. C. \& Norde, W. Statistical analysis of long- and short-range forces involved in bacterial adhesion to substratum surfaces as measured using atomic force microscopy. Appl. Environ. Microbiol. 77, 5065-5070, doi:10.1128/aem.00502-11 (2011).

28 Boks, N. P., Busscher, H. J., van der Mei, H. C. \& Norde, W. Bond-strengthening in staphylococcal adhesion to hydrophilic and hydrophobic surfaces using atomic force microscopy. Langmuir 24, 12990-12994 (2008).

29 Tollersrud, T., Berge, T., Andersen, S. R. \& Lund, A. Imaging the surface of Staphylococcus aureus by atomic force microscopy. APMIS 109, 541-545 (2001). 
30 Rukke, H. V., Hegna, I. K. \& Petersen, F. C. Identification of a functional capsule locus in Streptococcus mitis. Mol Oral Microbiol 27, 95-108, doi:10.1111/j.2041-1014.2011.00635.x (2012).

31 Wann, E. R., Dassy, B., Fournier, J. M. \& Foster, T. J. Genetic analysis of the cap5 locus of Staphylococcus aureus. FEMS Microbiol. Lett. 170, 97-103 (1999).

32 Kneidinger, B. et al. Three highly conserved proteins catalyze the conversion of UDP-N-acetyl-D-glucosamine to precursors for the biosynthesis of 0 antigen in Pseudomonas aeruginosa 011 and capsule in Staphylococcus aureus type 5. Implications for the UDP-N-acetyl-L-fucosamine biosynthetic pathway. J. Biol. Chem. 278, 3615-3627, doi:10.1074/jbc.M203867200 (2003).

33 Coldren, F. M. et al. Encapsulated Staphylococcus aureus strains vary in adhesiveness assessed by atomic force microscopy. Journal of Biomedical Materials Research. Part A 89, 402-410 (2009).

34 Chen, Y., Norde, W., van der Mei, H. C. \& Busscher, H. J. Bacterial cell surface deformation under external loading. MBio 3, doi:10.1128/mBio.00378-12 (2012).

35 Ovchinnikova, E. S., Krom, B. P., Busscher, H. J. \& van der Mei, H. C. Evaluation of adhesion forces of Staphylococcus aureus along the length of Candida albicans hyphae. BMC Microbiol. 12, 281, doi:10.1186/1471-2180-12-281 (2012).

$36 \mathrm{Xu}, \mathrm{C}$. P. et al. Staphylococcus aureus-fibronectin interactions with and without fibronectin-binding proteins and their role in adhesion and desorption. Appl. Environ. Microbiol. 74, 7522-7528, doi:10.1128/AEM.00948-08 (2008).

37 Chen, Y., Harapanahalli, A. K., Busscher, H. J., Norde, W. \& van der Mei, H. C. Nanoscale cell wall deformation impacts long-range bacterial adhesion forces on surfaces. Appl. Environ. Microbiol. 80, 637-643 (2014).

38 An, Y. H. \& Friedman, R. J. Concise review of mechanisms of bacterial adhesion to biomaterial surfaces. J. Biomed. Mater. Res. 43, 338-348 (1998).

39 Baselga, R., Albizu, I. \& Amorena, B. Staphylococcus aureus capsule and slime as virulence factors in ruminant mastitis. A review. Vet. Microbiol. 39, 195204 (1994).

40 Huo, K. et al. Osteogenic activity and antibacterial effects on titanium surfaces modified with Zn-incorporated nanotube arrays. Biomaterials 34, 34673478, doi:10.1016/j.biomaterials.2013.01.071 (2013).

41 Puckett, S. D., Taylor, E., Raimondo, T. \& Webster, T. J. The relationship between the nanostructure of titanium surfaces and bacterial attachment. Biomaterials 31, 706-713, doi:http://dx.doi.org/10.1016/j.biomaterials.2009.09.081 (2010).

42 Anselme, K. et al. The interaction of cells and bacteria with surfaces structured at the nanometre scale. Acta Biomater. 6, 3824-3846, doi:10.1016/j.actbio.2010.04.001 (2010).

43 Whitehead, K. A., Colligon, J. \& Verran, J. Retention of microbial cells in substratum surface features of micrometer and sub-micrometer dimensions. 
$\begin{array}{llll}\text { Colloids Surf. } \quad \text { B. } & \text { Biointerfaces }\end{array}$ doi:10.1016/j.colsurfb.2004.11.010 (2005).

44 Otto, M. Staphylococcal infections: mechanisms of biofilm maturation and detachment as critical determinants of pathogenicity. Annu. Rev. Med. 64, 175-188, doi:10.1146/annurev-med-042711-140023 (2013). 